\title{
Tetris Tight Frames Construction via Hadamard Matrices
}

\author{
A. Abdollahi and M. Monfaredpour \\ Department of Mathematics, College of Sciences, Shiraz University, Shiraz 71454, Iran \\ Correspondence should be addressed to A. Abdollahi; abdollahi@shirazu.ac.ir
}

Received 30 April 2014; Accepted 23 September 2014; Published 27 October 2014

Academic Editor: Hossein Jafari

Copyright (C) 2014 A. Abdollahi and M. Monfaredpour. This is an open access article distributed under the Creative Commons Attribution License, which permits unrestricted use, distribution, and reproduction in any medium, provided the original work is properly cited.

We present a new method to construct unit norm tight frames by applying altered Hadamard matrices. Also we determine an elementary construction which can be used to produce a unit norm frame with prescribed spectrum of frame operator.

\section{Introduction and Preliminaries}

Frames were first introduced in 1952 by Duffin and Schaeffer [1] in the context of nonharmonic Fourier series. They are system of functions in Hilbert spaces that provide numerically stable methods for finding overcomplete decompositions of vectors and such are useful tools in various signal processing applications, data compression, wireless communications, and so on [2-4]. Frames in finite dimensional Hilbert spaces have become of interests for many of researches $[5,6]$. One of the important subjects in this area is the way for constructing such frames. Some methods of construction finite tight frames are stated by researchers [7-9].

Let $N$ be a positive integer. A sequence of vectors $\left\{f_{i}\right\}_{i \in I}$ in Hilbert space $\mathbb{R}^{N}$ is said to be a frame (see also [10]) for $\mathbb{R}^{N}$ if there exist constants $A$ and $B$ such that $0<A \leq B<\infty$ and

$$
A\|f\|^{2} \leq \sum_{i \in I}\left|\left\langle f, f_{i}\right\rangle\right|^{2} \leq B\|f\|^{2} \quad \forall f \in \mathbb{R}^{N} .
$$

The numbers $A$ and $B$ are called frame bounds and they are not unique. The frame $\left\{f_{i}\right\}_{i \in I}$ is said to be tight (or $A$-tight) if $A=B$. In this case, $A$ is said to be the frame constant and it is a Parsval frame, if $A=B=1$. When the index set $I$ is a finite set, the frame will be called finite. A normalized frame or unit norm frame is the one in which elements have the norm one.

If only the right side of the inequalities (1) holds, then $\left\{f_{i}\right\}_{i \in I}$ is called a Bessel sequence and if $\left\{f_{i}\right\}_{i \in I}$ is a normalized Bessel sequence in $\mathbb{R}^{N}$, then $\left\{f_{i}\right\}_{i \in I}$ is finite sequence [5]. According to this, our frame will be the form $\left\{f_{i}\right\}_{i=1}^{M}$ whenever
$M$ is some positive integer. Also we will replace $l^{2}(I)$ by $\mathbb{R}^{M}$. To each Bessel sequence $\left\{f_{i}\right\}_{i=1}^{M}$ corresponds an operator

$$
T: \mathbb{R}^{N} \longrightarrow \mathbb{R}^{M} \quad T(f)=\left\{\left\langle f, f_{i}\right\rangle\right\}_{i=1}^{M}
$$

called analysis operator which is well-defined and bounded operator. Its adjoint is the operator

$$
T^{*}: \mathbb{R}^{M} \longrightarrow \mathbb{R}^{N} \quad T^{*}\left(\left\{c_{i}\right\}_{i=1}^{M}\right)=\sum_{i=1}^{M} c_{i} f_{i},
$$

called the synthesis operator. If $\left\{f_{i}\right\}_{i=1}^{M}$ is a frame with frame bounds $A$ and $B$, then the operator

$$
T^{*} T: \mathbb{R}^{N} \longrightarrow \mathbb{R}^{N}, \quad T^{*} T(f)=\sum_{i=1}^{M}\left\langle f, f_{i}\right\rangle f_{i}
$$

is called the frame operator of the frame $\left\{f_{i}\right\}_{i=1}^{M}$. It is a positive, self-adjoint, bounded, and hence invertible operator with the inverse $\left(T^{*} T\right)^{-1}$. Benedetto and Fickus in [5] have proved that if $\left\{f_{i}\right\}_{i=1}^{M}$ is a normalized $A$-tight frame for a $N$-dimensional Hilbert space $\mathbb{H}$, then $A=M / N$.

In this paper, we address the question of how to efficiently construct unit norm tight frame. After reviewing known results about the Spectral Tetris Construction and introducing the Spectral Hadamard-Tetris Construction in Section 2, our main result in Section 3 presents a way to construct unit norm tight frames that we call HadamardTetris Construction. In Section 4, we introduce another 
version of Hadamard-Tetris which can be used to construct unit norm frames with different eigenvalues for the frame operator. We give necessary condition for this version of Hadamard-Tetris to produce the desired frames.

\section{Special Hadamard-Tetris Construction}

In [11] by using the Schur-Horn Theorem, the authors have shown how to construct every possible frame in which frame operator has a given spectrum and in which vectors are of given prescribed norms, but their method is complex. In [12], the authors have presented another construction of unit norm frames which is called "Spectral Tetris Construction" (STC). Spectral Tetris is a flexible and elementary method to construct unit norm frames with a given frame operator, having all of its eigenvalues greater than or equal to two. But that way is limited to the case of frames with $M$ elements for $\mathbb{R}^{N}$ in which $M \geq 2 N$. However, they extended the existing construction to unit norm tight frame of redundancy less than two [13], but those frames are in $\mathbb{C}^{N}$ not in $\mathbb{R}^{N}$.

In this paper, we implement an algorithm which is named Hadamard-Tetris for the construction of unit norm tight frames. Hadamard-Tetris constructs synthesis matrix $N \times$ $M$ with unit norm columns in which rows are pairwise orthogonal and square sum to $M / N$. By this construction, the frame operator is $(M / N) I$. The main advantage here is given an elementary and easily implementable algorithm for constructing frames.

In our way, the assumption on the spectrum of the unit norm tight frame $(M / N \geq 2)$ in STC can be dropped and construct unit norm tight frame for $\mathbb{R}^{N}$ with $M$ elements, where $M$ and $N$ are positive integers and satisfy the following condition.

If we can decompose $M$ as $K=M-N+1$ summation $d_{i}(1 \leq i \leq K)$, that is, $M=\sum_{i=1}^{K} d_{i}$, which there exist Hadamard matrices of sizes $d_{i}$ 's and they satisfy the condition

$$
(i-1) \frac{M}{M-N} \leq \sum_{j=1}^{i} d_{j}<i \frac{M}{M-N} \quad 1 \leq i \leq K-1
$$

then there exists a unit norm tight frame for $\mathbb{R}^{N}$ with sparsity at $\operatorname{most} \sum_{j=1}^{K} d_{j}^{2}$.

A frame constructed via the Hadamard-Tetris construction is called Hadamard-Tetris frame.

Aside from the fact that Hadamard-Tetris frames are easy to construct, their major advantage for applications is the sparsity of their synthesis matrices. This sparsity is dependent on the decomposition of $M$.

In our construction for any positive integers $M$ and $N(M>N)$, we put $K=M-N+1$ and if we could decompose $M$ as $K$ summation of power of two, then we give a unit normalized tight frame for $\mathbb{R}^{N}$ with $M$ elements. Hence $K$ has minimum value. If $N=\sum_{\{i=0\}}^{t} a_{i} 2^{i}$ is the binary representation of $N$, where $a_{i}$ 's are 0 or 1 , then $\sum_{\{i=0\}}^{t} a_{i} \leq K$.
Parameter:

\section{HTC(I): Hadamard-Tetris construction}

(i) Dimension $N \in \mathbb{N}$.

Algorithm:

(1) For $j=1$ to $N-1$

(2) $\quad x_{j}=1-\frac{j}{N}$.

(3) $\quad f_{2 j-1}=\sqrt{x_{j}} e_{j}+\sqrt{1-x_{j}} e_{j+1}$.

(4) $\quad f_{2 j}=\sqrt{x_{j}} e_{j}-\sqrt{1-x_{j}} e_{j+1}$.

(5) end.

Output:

(i) unit norm tight frame $\left\{f_{j}\right\}_{j=1}^{2 N-2}$ for $\mathbb{R}^{N}$.

Algorithm 1: The HTC(I) algorithm for constructing a unit norm tight frame with $(2 N-2)$-elements for $\mathbb{R}^{N}$.

In special case, when $M=2 N-2$, we can construct synthesis matrix $T^{*}$ of size $N \times M$ which has $f_{i}$ 's as its columns as in Algorithm 1.

Theorem 1. Let $N$ be a positive integer number. Then the sparsest synthesis matrix of the $(2 N-2)$-element HadamardTetris unit norm tight frame for $\mathbb{R}^{N}$ which consists of $N-1$ blocks of size 2 can be constructed by HTC(I) and its sparsity is $4(N-1)$.

Proof. Let $M=2 N-2$. By the construction of HTC(I), in lines (3) and (4), $f_{i}$ 's are normalized. We should show that the square norm of each row of synthesis matrix is $M / N$. It is clear that the square norm of the first row of the matrix is $M / N$ and, in the last row, since $x_{N-1}=1 / N$, the square norm of the last row is

$$
2\left(1-\frac{1}{N}\right)=\frac{2 N-2}{N}=\frac{M}{N}
$$

Also, for $2 \leq j \leq N-1$, the square norm of $j$ th row is

$$
\begin{aligned}
2\left(1-x_{j-1}\right)+2 x_{j} & =2\left(\frac{j-1}{N}\right)+2\left(1-\frac{j}{N}\right) \\
& =\frac{2 N-2}{N}=\frac{M}{N} .
\end{aligned}
$$

In this construction, synthesis matrix of frame consists of $N-1$ blocks of size 2 , so the sparsity of the matrix is $4(N-$ 1).

In [14], Casazza et al. proved that the algorithm STC can be performed to generate a unit norm tight frame of $M$ vectors in $\mathbb{R}^{N}$ if and only if $\lambda:=M / N$ or $\lambda$ is of the form $\lambda=(2 L-1) / L$, for some positive integer $L$. Whereas we exhibit an algorithm that utilizes matrices of size 2 such as

$$
\left[\begin{array}{cc}
\sqrt{x} & \sqrt{x} \\
\sqrt{1-x} & -\sqrt{1-x}
\end{array}\right]
$$


used in the construction of STC. For example, for $M=4$ and $N=3$, the matrix

$$
\left[\begin{array}{cccc}
\sqrt{\frac{2}{3}} & \sqrt{\frac{2}{3}} & 0 & 0 \\
\sqrt{\frac{1}{3}} & -\sqrt{\frac{1}{3}} & \sqrt{\frac{1}{3}} & \sqrt{\frac{1}{3}} \\
0 & 0 & \sqrt{\frac{2}{3}} & -\sqrt{\frac{2}{3}}
\end{array}\right]
$$

is synthesis matrix of $\mathbb{R}^{3}$.

Example 2. If $N=4$ and $M=6$, then

$$
\left[\begin{array}{cccccc}
\sqrt{\frac{3}{4}} & \sqrt{\frac{3}{4}} & 0 & 0 & 0 & 0 \\
\sqrt{\frac{1}{4}} & -\sqrt{\frac{1}{4}} & \sqrt{\frac{1}{2}} & \sqrt{\frac{1}{2}} & 0 & 0 \\
0 & 0 & \sqrt{\frac{1}{2}} & -\sqrt{\frac{1}{2}} & \sqrt{\frac{1}{4}} & \sqrt{\frac{1}{4}} \\
0 & 0 & 0 & 0 & \sqrt{\frac{3}{4}} & -\sqrt{\frac{3}{4}}
\end{array}\right]
$$

is synthesis of 6-elements for $\mathbb{R}^{4}$.

\section{Hadamard-Tetris Frame}

In this section we provide a new method for constructing finite normalized tight frames (FNTFs). In brief, we want to construct $N \times M$ synthesis matrix $T^{*}$ which has

(i) columns of unit norm;

(ii) orthogonal rows, meaning that the frame operator $T^{*} T$ is diagonal;

(iii) rows of constant norm, meaning that $T^{*} T$ is a constant multiple of the identity matrix.

The Hadamard-Tetris Construction (HTC) is capable of constructing unit norm tight frames, with the number of elements that decompose as $d_{i}(1 \leq i \leq M-N+$ $1)$. It constructs the synthesis matrices of such frames by successively filling of size $d_{i}$ of $M-N+1$ blocks. In this construction, we use altered Hadamard matrices.

The importance of Hadamard matrices to our construction stems from the fact that they have orthogonal rows and columns and that all entries have the same modulus. In the course of the construction, we will have to alter the row norms of the Hadamard matrices by multiplying rows with appropriate constants. While this will destroy the pairwise orthogonality of the columns, it will preserve the pairwise orthogonality of the rows, which is the crucial feature for our construction to work.
Definition 3. Given positive integers $M$ and $N$, we denote a matrix by $H_{d}$ or call it a $H_{d}$ block, if it is derived from a Hadamard matrix of size $d$ by multiplying the entries of the $j$ th row of Hadamard matrix by $\sqrt{M / N d}$ for $j=2, \ldots, d-1$ and the entries of the first and the last row of Hadamard matrix by $r_{1}$ and $r_{d}$ and if it has normalized columns. We call $r_{1}$ and $r_{d}$ the first and the last correction factor of $H_{d}$, respectively.

Note that the row norm of a $H_{d}$ block equals $\sqrt{M / N}$, except possibly for the first and the last rows. We want to present an algorithm following the lines of Example 4; that is, we want to compose the desired synthesis matrix which consists of square blocks in which first and last rows successively overlap. If we use $K$ square matrix as building blocks of the $N \times M$ synthesis matrix, then, due to the overlapping, we have $M=N+(K-1)$.

It is perhaps most instructive to first look at the example of the construction that we are going to introduce in this section.

Example 4. We construct a 6-elements unit norm tight frame in $\mathbb{R}^{5}$. We can start filling the desired $5 \times 6$ synthesis matrix with an altered $4 \times 4$ Hadamard matrix in the upper left corner. The alteration we make is to multiply the entries of the first row by $\sqrt{3 / 10}$ in size to make the first row have the desired norm $\sqrt{6 / 5}$. We multiply the second and the third rows of $4 \times 4$ Hadamard matrix by $\sqrt{3 / 10}$ to make the norm of those equal to $\sqrt{6 / 5}$.

To get normalized column, we multiply the forth row of the $4 \times 4$ Hadamard matrix by $\sqrt{1 / 10}$. At this point, we have constructed the first three rows and the first four columns of the desired synthesis matrix:

$$
\left[\begin{array}{cccccc}
\sqrt{\frac{3}{10}} & \sqrt{\frac{3}{10}} & -\sqrt{\frac{3}{10}} & \sqrt{\frac{3}{10}} & 0 & 0 \\
-\sqrt{\frac{3}{10}} & \sqrt{\frac{3}{10}} & -\sqrt{\frac{3}{10}} & -\sqrt{\frac{3}{10}} & 0 & 0 \\
\sqrt{\frac{3}{10}} & -\sqrt{\frac{3}{10}} & -\sqrt{\frac{3}{10}} & -\sqrt{\frac{3}{10}} & 0 & 0 \\
\sqrt{\frac{1}{10}} & \sqrt{\frac{1}{10}} & \sqrt{\frac{1}{10}} & -\sqrt{\frac{1}{10}} & . & . \\
0 & 0 & 0 & 0 & .
\end{array}\right] .
$$

Note that, so far, we have constructed a matrix in which first four rows are orthogonal, no matter how we keep filling the first four rows. The fourth row at this point has norm $\sqrt{2 / 5}$, while we need to make it have norm $\sqrt{6 / 5}$.

We can insert altered $2 \times 2$ Hadamard matrix in the same fashion as above. To do this, we would multiply its first row by the factor $\sqrt{2 / 5}$ in size to have the forth row of the synthesis 
matrix square sum to $6 / 5$ and its second row by the factor $\sqrt{3 / 5}$ to get

$$
\left[\begin{array}{cccccc}
\sqrt{\frac{3}{10}} & \sqrt{\frac{3}{10}} & -\sqrt{\frac{3}{10}} & \sqrt{\frac{3}{10}} & 0 & 0 \\
-\sqrt{\frac{3}{10}} & \sqrt{\frac{3}{10}} & -\sqrt{\frac{3}{10}} & -\sqrt{\frac{3}{10}} & 0 & 0 \\
\sqrt{\frac{3}{10}} & -\sqrt{\frac{3}{10}} & -\sqrt{\frac{3}{10}} & -\sqrt{\frac{3}{10}} & 0 & 0 \\
\sqrt{\frac{1}{10}} & \sqrt{\frac{1}{10}} & \sqrt{\frac{1}{10}} & -\sqrt{\frac{1}{10}} & \sqrt{\frac{2}{5}} & -\sqrt{\frac{2}{5}} \\
0 & 0 & 0 & 0 & \sqrt{\frac{3}{5}} & \sqrt{\frac{3}{5}}
\end{array}\right] .
$$

The latter matrix is the synthesis matrix of the desired frame, since its columns are normalized and its rows are pairwise orthogonal and square sum to $6 / 5$.

The next theorem is the main theorem of this section.

Theorem 5 (main theorem). Let $M$ and $N(M>N)$ be positive integers and $K=M-N+1$. Furthermore, assume that $M$ can be decomposed as $M=\sum_{j=1}^{K} d_{j}$ such that, for each $1 \leq i \leq K-1$,

$$
(i-1) \frac{M}{M-N} \leq \sum_{j=1}^{i} d_{j}<i \frac{M}{M-N},
$$

and there exist Hadamard matrices of sizes $d_{i}$ 's. Then HTC(II) gives a unit normalized tight frame for $\mathbb{R}^{N}$ with $M$ elements.

In special case, when $M-N=1$, then the condition on $d_{i}$ 's is confirmed automatically. Indeed, if $d_{1}$ is selected such that $0<d_{1}<M$, then $0<d_{2}=M-d_{1}<M$.

Corollary 6. If $M=d_{1}+d_{2}$ such that there exist Hadamard matrices of sizes $d_{1}$ and $d_{2}$, then the synthesis matrix of the $M$ elements for $\mathbb{R}^{M-1}$ can be constructed via HTC(II).

To prove Theorem 5, we need to collect some information about the first and the last correction factors of $H_{d_{i}}$ 's.

In construction of synthesis matrix of size $N \times M$, we use $K=M-N+1$ blocks of sizes $d_{i}$ 's $(1 \leq i \leq K)$, where $M=$ $\sum_{i=1}^{K} d_{i}$.

As was mentioned, each block is $H_{d_{i}}$ that derived from the Hadamard matrix of size $d_{i}$. If the factor that multiplies the entries of the $j$ th row of Hadamard matrix of size $d_{i}$ is shown by $r_{j, i}$, where $1 \leq j \leq d_{i}$, then, for each block of size $d_{i}$, the correction factor is

$$
r_{j, i}^{2}=\frac{M}{N d_{i}} \quad\left(2 \leq j \leq d_{i}-1\right) .
$$

The last row of a block and the first row of the following block appear in the same row of the synthesis matrix and while the last correction factor is chosen to ensure normalized columns, the following first correction factor is chosen to guarantee that the rows of the synthesis matrix have square norm $M / N$.

Hence, we have the following relationships for $r_{1, i}$ and $r_{d_{i}, i}$, where $1 \leq i \leq K$.

Lemma 7. If $r_{1, i}$ and $r_{d_{i}, i}$ are the first and the last correction factors, respectively, then

$$
\begin{aligned}
& r_{d_{i}, i}^{2}=1-r_{1, i}^{2}-\frac{M}{N}+2 \frac{M}{N d_{i}}, \\
& r_{1, i+1}^{2}=\frac{1}{d_{i+1}}\left(\frac{M}{N}-d_{i} r_{d_{i}, i}^{2}\right) .
\end{aligned}
$$

Proof. The correction factors of second row to $\left(d_{i}-1\right)$ th row of $H_{d_{i}}$ are

$$
r_{j, i}=\sqrt{\frac{M}{N d_{i}}} \quad\left(2 \leq j \leq d_{i}-1\right) .
$$

Since norm of each column of $H_{d_{i}}$ must be one, we have

$$
r_{1, i}^{2}+\left(d_{i}-2\right) \frac{M}{N d_{i}}+r_{d_{i}, i}^{2}=1
$$

and so on

$$
r_{d_{i}, i}^{2}=1-r_{1, i}^{2}-\frac{M}{N}+2 \frac{M}{N d_{i}}
$$

Also the last correction factor of $H_{d_{i}}$ and the first correction factor of $H_{d_{i+1}}$ must be chosen such that the square norm of that row is $M / N$. Hence

$$
d_{i} r_{d_{i}, i}^{2}+d_{i+1} r_{1, i+1}^{2}=\frac{M}{N}
$$

and so on

$$
r_{1, i+1}^{2}=\frac{1}{d_{i+1}}\left(\frac{M}{N}-d_{i} r_{d_{i}, i}^{2}\right) .
$$

By combining formulas (15) in Lemma 7, we get the following recursive relations:

$$
\begin{aligned}
& r_{1, i+1}^{2}=\frac{d_{i}}{d_{i+1}}\left(\frac{M}{N}+r_{1, i}^{2}-1\right)-\frac{M}{N d_{i+1}} \\
& r_{d_{i+1}, i+1}^{2}=\frac{d_{i}}{d_{i+1}} r_{d_{i}, i}^{2}-\frac{M-N}{N}+\frac{M}{N d_{i+1}}
\end{aligned}
$$

for $1 \leq i \leq K-1$.

Lemma 8. If $r_{1, i}$ and $r_{d_{i}, i}$ are the first and the last correction factors of $H_{d_{i}}$, respectively, then

$$
\begin{gathered}
r_{1, i}^{2}=\frac{1}{N d_{i}}\left((M-N) \sum_{j=1}^{i-1} d_{j}-(i-2) M\right) \quad(i \geq 2), \\
r_{d_{i}, i}^{2}=\frac{1}{N d_{i}}\left(i M-(M-N) \sum_{j=1}^{i} d_{j}\right) \quad(i \geq 1) .
\end{gathered}
$$


Proof. We prove these by induction on $i$. The first steps of the induction are trivially true. Assume that the identities are true for $i$. For the case $i+1$, the following identities hold by using formulas (21) and the induction hypothesis:

$$
\begin{aligned}
& r_{1, i+1}^{2}=\frac{d_{i}}{d_{i+1}}\left(\frac{M}{N}+r_{1, i}^{2}-1\right)-\frac{M}{N d_{i+1}} \\
& =\frac{d_{i}}{d_{i+1}}\left(\frac{M}{N}+\left(\frac{\sum_{j=1}^{i-1} d_{j}}{d_{i}}\right)\left(\frac{M-N}{N}\right)\right. \\
& \left.-\left(\frac{i-2}{d_{i}}\right)\left(\frac{M}{N}\right)-1\right)-\frac{M}{N d_{i+1}} \\
& =\frac{1}{N d_{i+1}}\left(d_{i} M+(M-N) \sum_{j=1}^{i-1} d_{j}\right. \\
& \left.-(i-2) M-N d_{i}-M\right) \\
& =\frac{1}{N d_{i+1}}\left((M-N) \sum_{j=1}^{i} d_{j}-(i-1) M\right), \\
& r_{d_{i+1}, i+1}^{2}=\frac{d_{i}}{d_{i+1}} r_{d_{i}, i}^{2}-\frac{M-N}{N}+\frac{M}{N d_{i+1}} \\
& =\frac{d_{i}}{d_{i+1}}\left(\left(\frac{i}{d_{i}}\right)\left(\frac{M}{N}\right)-\left(\frac{\sum_{j=1}^{i} d_{j}}{d_{i}}\right)\left(\frac{M-N}{N}\right)\right) \\
& -\frac{M-N}{N}+\frac{M}{N d_{i+1}} \\
& =\frac{1}{N d_{i+1}}\left(i M-(M-N) \sum_{j=1}^{i} d_{j}\right. \\
& \left.-d_{i+1}(M-N)+M\right) \\
& =\frac{1}{N d_{i+1}}\left((i+1) M-(M-N) \sum_{j=1}^{i+1} d_{j}\right) \text {. }
\end{aligned}
$$

Now we are ready to prove the main Theorem 5.

Proof. We check that synthesis matrix constructed by HTC(II) has row norm equal $\sqrt{M / N}$ and columns are normalized. First, we show that each row of synthesis matrix, which is constructed via HTC(II), has square norm $M / N$. It is enough to show that the last row of $H_{d_{i}}$ together with the first row of $H_{d_{i+1}}$ makes square norm $M / N$. In other words,

$$
d_{i} r_{d_{i}, i}^{2}+d_{i+1} r_{1, i+1}^{2}=\frac{M}{N}
$$

By Lemma 8, we have

$$
\begin{aligned}
d_{i} r_{d_{i}, i}^{2}+d_{i+1} r_{1, i+1}^{2}= & \frac{1}{N}\left(i M-(M-N) \sum_{j=1}^{i} d_{j}\right. \\
& \left.\quad+(M-N) \sum_{j=1}^{i} d_{j}-(i-1) M\right) \\
= & \frac{M}{N} .
\end{aligned}
$$

Now, we show each column of synthesis matrix that is constructed with HTC(II) has norm 1; that is,

$$
r_{1, i}^{2}+\left(d_{i}-2\right) \frac{M}{N d_{i}}+r_{d_{i}, i}^{2}=1
$$

By Lemma 8, we have

$$
\begin{aligned}
r_{1, i}^{2}+r_{d_{i}, i}^{2}= & \frac{1}{N d_{i}}\left((M-N)\left(\sum_{j=1}^{i-1} d_{j}-\sum_{j=1}^{i} d_{j}\right)\right. \\
& \quad+M(-i+2+i)) \\
= & -\left(\frac{M-N}{N}\right)+2 \frac{M}{N d_{i}} \\
= & 1-\left(d_{i}-2\right) \frac{M}{N d_{i}} .
\end{aligned}
$$

We have to ensure that the last correction factor of the final block inserted into the synthesis matrix equals $\sqrt{M / N d_{K}}$ (in other words, the next first correction factor would be zero, but we have arrived at this point where the algorithm terminates).

In Algorithm 2, line (9), when $i:=K$ and $t:=d_{K}$, we have

$$
r_{d_{K}, K}=\sqrt{\frac{1}{N d_{K}}\left(K M-(M-N) \sum_{j=1}^{K} d_{j}\right)}=\sqrt{\frac{M}{N d_{K}}}
$$

and in line (4), for $i:=K+1$, we have $r_{1, d_{K+1}}=0$.

Note that if we do a HTC(II) as in Example 4 with $K$ altered Hadamard matrices of sizes $d_{1}, \ldots, d_{K}$, then the sparsity of the synthesis matrix is $\sum_{i=1}^{K} d_{i}^{2}$.

The next lemma states that if we construct synthesis matrix with blocks of sizes $d_{1}, d_{2}, \ldots, d_{K}$, each condition on $d_{i}$ must be established for $d_{K-i+1}$ and vice versa.

Lemma 9. Let $M$ and $N$ be positive integers with $M>N$, $K=M-N+1$, and $M=\sum_{j=1}^{K} d_{j}$ such that $d_{j}$ 's are satisfied in inequalities:

$$
\begin{array}{r}
(i-1) \frac{M}{M-N}<\sum_{j=1}^{i} d_{j}<i \frac{M}{M-N} \\
(0 \leq i \leq K-1) .
\end{array}
$$


Parameters:

\section{HTC(II): Hadamard-Tetris Construction}

(i) Dimension $N \in \mathbb{N}$.

(ii) Number of frame elements $M \in \mathbb{N}$ such that decompose as $M=\sum_{i=1}^{K} d_{i}$, where $K=M-N+1$.

(iii) Hadamard matrices, $H_{d_{i}}=\left[a_{k j}^{\left(d_{i}\right)}\right]_{1 \leq k, j \leq d_{i}}$ of size $d_{i}$ for $i=1, \ldots, K$.

\section{Algorithm:}

(1) $s=0, m=0$.

(2) For $i=1$ to $K$ do

(3) For $t=1$ to $d_{i}$ do

end.

$$
\begin{aligned}
& r_{1, i}=\sqrt{\frac{1}{N d_{i}}\left((M-N) \sum_{j=1}^{i-1} d_{j}-(i-2) M\right) .} \\
& r_{1,1}=\sqrt{\frac{M}{N d_{1}}} .
\end{aligned}
$$

For $j=2$ to $d_{i}-1$ do

$$
r_{j, i}=\sqrt{\frac{M}{N d_{i}}}
$$

(10) $\quad f_{t+m}=\sum_{j=1}^{d_{i}} a_{t j}^{\left(d_{i}\right)} r_{j, i} e_{s+j}$.

(11) end.

(12) $m=m+d_{i}$.

(13) $s=s+d_{i}-1$.

(14) end.

(i) unit norm tight frame $\left\{f_{j}\right\}_{j=1}^{M}$ for $\mathbb{R}^{N}$.

Algorithm 2: The HTC(II) algorithm for constructing a unit norm tight frame with $M$ elements for $\mathbb{R}^{N}$.

Then

$$
\begin{array}{r}
(i-1) \frac{M}{M-N}<\sum_{j=1}^{i} d_{K-j+1}<i \frac{M}{M-N} \\
(0 \leq i \leq K-1) .
\end{array}
$$

Proof. Since $\sum_{j=1}^{K} d_{j}=M$, so

$$
\sum_{j=1}^{i} d_{K-j+1}=\sum_{j=1}^{K} d_{j}-\sum_{j=1}^{K-i} d_{j}=M-\sum_{j=1}^{K-i} d_{j}
$$

In the other hand, by replacing $i$ with $K-i$ in the inequality (30), we have

$$
\frac{M}{M-N}(K-i-1)<\sum_{j=1}^{K-i} d_{j}<(K-i) \frac{M}{M-N}
$$

and so on

$$
\begin{aligned}
M-(K-i) \frac{M}{M-N}< & M-\sum_{j=1}^{K-i} d_{j}<M-\frac{M}{M-N} \\
& \times(K-i-1) .
\end{aligned}
$$

Hence, we have

$$
\begin{aligned}
\frac{M}{M-N}(M-N+i-K)< & \sum_{j=1}^{i} d_{K-j+1}<\frac{M}{M-N} \\
& \times(M-N-K+i+1),
\end{aligned}
$$

which give inequalities (31).

Following Lemma 9, if $T^{*}=\left[a_{i j}\right]$ is a synthesis matrix of size $N \times M$, then $F^{*}=\left[b_{i j}\right]$, where $b_{i j}=a_{(N-i+1)(M-j+1)}$, is also having the same situation.

Since Hadamard matrices of the size power of 2 exist, so we use altered Hadamard matrices of size $2^{s_{i}}$, where

$$
2^{s_{i}}=\max \left\{2^{s} \mid(i-1) \frac{M}{M-N} \leq \sum_{j=1}^{i-1} 2^{s_{j}}+2^{s}<i \frac{M}{M-N}\right\},
$$

for $1 \leq i \leq K-1$. However, it is provided that there exists a Hadamard matrix of size $d_{K} \times d_{K}$, with $d_{K}=M-\sum_{j=1}^{K-1} 2^{s_{j}}$. In this case, the algorithm HTC builds the synthesis matrix of the tight frame as in Example 4 by inserting $H_{d_{i}}$, where $d_{i}=$ $2^{s_{i}}(1 \leq i \leq K-1)$ and $d_{K}=M-\sum_{j=1}^{K-1} 2^{s_{j}}$. 


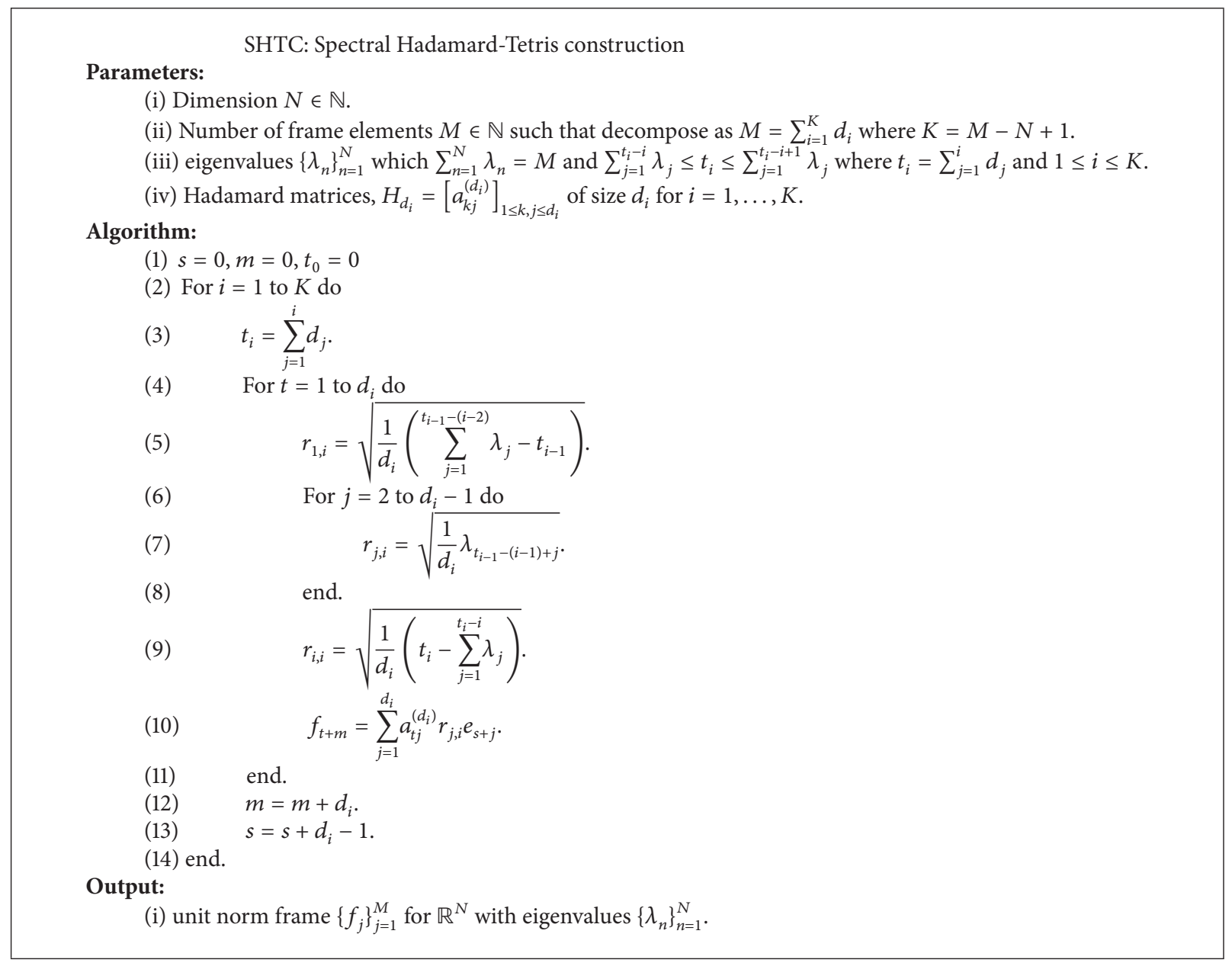

Algorithm 3: The SHTC algorithm for constructing a unit norm tight frame with $M$ elements for $\mathbb{R}^{N}$.

\section{Spectral Hadamard Tetris Construction}

Another case of HTC is the case of unit norm but not necessarily tight frames. Such frames are known to exist, provided that the eigenvalues of the frame operator sum up to the number of frame vectors [15]. In this section, we give a version of Hadamard-Tetris Construction which uses altered Hadamard matrices to construct unit norm frames with a given spectrum of frame operator. Our method cannot construct all such frames. We give necessary condition under which this construction works. To construct unit norm frame with prescribed spectrum, we are looking for a matrix with columns square summing up to 1 and rows square summing up to $\left\{\lambda_{n}\right\}_{n=1}^{N}$. In Algorithm 3, we present a modified Hadamard-Tetris algorithm HTC, allowing the construction of such frames.

Given a fixed dimension $N$ and frame cardinality $M$, a necessary condition on the prescribed eigenvalues $\left\{\lambda_{n}\right\}_{n=1}^{N}$ of the frame operator for SHTC to work is given in the following theorem.

Theorem 10. Let $\sum_{n=1}^{N} \lambda_{n}=M=\sum_{i=1}^{K} d_{i}$, where $K=M-$ $N+1$, and there exist Hadamard matrices of sizes $d_{i}$ 's. Then SHTC can be used to produce unit norm frame for $\mathbb{R}^{N}$ with eigenvalues $\left\{\lambda_{n}\right\}_{n=1}^{N}$ if there are some permutations of $\left\{\lambda_{n}\right\}_{n=1}^{N}$ and $\left\{d_{i}\right\}_{i=1}^{K}$ such that, for each $i=1, \ldots, K$, we have

$$
\sum_{j=1}^{t_{i}-i} \lambda_{j} \leq t_{i} \leq \sum_{j=1}^{t_{i}-i+1} \lambda_{j},
$$

where $t_{i}=\sum_{j=1}^{i} d_{j}$.

Here, we present an example in which STC does not work whereas SHTC works.

Example 11. There are choices of prescribed eigenvalues which satisfy the conditions of Theorem 10 that unit norm frame with given eigenvalues exists, but STC cannot be used to construct such a frame because no rearrangement of eigenvalues satisfies in their conditions. An example of this kind is $\left\{\lambda_{n}\right\}_{n=1}^{4}=\{0.4,2.4,1.1,1.1\}$ as eigenvalues of frame with 5 elements for $\mathbb{R}^{4}$.

Sometimes, one rearrangement of eigenvalues and dimensions of Hadamard matrices is satisfied in desired condition while another is not. For example, the SHTC cannot be performed for the sequence of $\lambda_{i}$ 's is the given 
rearrangement $0.4,1.1,1.1,2.4$ and $d_{1}=4, d_{2}=1$. However, rearranging the eigenvalues to the rearrangement $0.4,2.4,1.1,1.1$ allows SHTC to construct the desired frame. In this case, its synthesis matrix will be

$$
\left[\begin{array}{ccccc}
\sqrt{\frac{1}{10}} & \sqrt{\frac{1}{10}} & -\sqrt{\frac{1}{10}} & \sqrt{\frac{1}{10}} & 0 \\
-\sqrt{\frac{6}{10}} & \sqrt{\frac{6}{10}} & -\sqrt{\frac{6}{10}} & -\sqrt{\frac{6}{10}} & 0 \\
\sqrt{\frac{11}{40}} & -\sqrt{\frac{11}{40}} & -\sqrt{\frac{11}{40}} & -\sqrt{\frac{11}{40}} & 0 \\
\sqrt{\frac{1}{40}} & \sqrt{\frac{1}{40}} & \sqrt{\frac{1}{40}} & -\sqrt{\frac{1}{40}} & 1
\end{array}\right]
$$

So, there is no reason that SHTC works when the eigenvalues are in the monotonic rearrangement.

Note that since we make no assumption about the ordering of the sequences $\left\{\lambda_{n}\right\}_{n=1}^{N}$ and $\left\{d_{i}\right\}_{i=1}^{K}$, we may permute the elements of the sequences in order to make them ready to satisfy in the inequality (37).

Given sequences of eigenvalues and $\left\{d_{i}\right\}_{i=1}^{K}$, where $K=$ $M-N+1$ and there exist Hadamard matrices of sizes $d_{i}$ 's, it may be time-consuming to find permutation of these sequences which satisfy in Theorem 10. Until now, we could not find simple conditions on these sequences that SHTC can be performed. It has been a mystery as to when Hadamard Tetris works and when fails.

\section{Conflict of Interests}

The authors declare that there is no conflict of interests regarding the publication of this paper.

\section{Acknowledgments}

The authors are grateful to Professor Hossein Jafari, the Editor of AAA, and the referee for their valuable comments.

\section{References}

[1] R. J. Duffin and A. C. Schaeffer, "A class of nonharmonic Fourier series," Transactions of the American Mathematical Society, vol. 72, pp. 341-366, 1952.

[2] J. Kovačević and A. Chebira, "Life beyond bases: the advent of frames (Parts I and II)," IEEE Signal Processing Magazine, vol. 24, no. 5, pp. 115-125, 2007.

[3] E. Šoljanin, "Tight frames in quantum information theory," in Proceedings of the DIMACS Workshop on Source Coding and Harmonic Analysis, Rutgers, NJ, USA, May 2002.

[4] T. Strohmer and J. Heath, "Grassmannian frames with applications to coding and communication," Applied and Computational Harmonic Analysis, vol. 14, no. 3, pp. 257-275, 2003.

[5] J. J. Benedetto and M. Fickus, "Finite normalized tight frames," Advances in Computational Mathematics, vol. 18, no. 2-4, pp. 357-385, 2003.

[6] N. Cotfas and J. P. Gazeau, "Finite tight frames and some applications," Journal of Physics, A: Mathematical and Theoretical, vol. 43, no. 19, Article ID 193001, 2010.
[7] D. F. Li and W. C. Sun, "Expansion of frames to tight frames," Acta Mathematica Sinica (English Series), vol. 25, no. 2, pp. 287292, 2009.

[8] J. C. Tremain, "Algorithmic constructions of unitary matrices and tight frames," http://arxiv.org/abs/1104.4539vl.

[9] D.-J. Feng, L. Wang, and Y. Wang, "Generation of finite tight frames by Householder transformations," Advances in Computational Mathematics, vol. 24, no. 1-4, pp. 297-309, 2006.

[10] O. Christensen, Frames and Bases, An Introductory Course, Birkhäuser, Boston, Mass, USA, 2008.

[11] J. Cahill, M. Fickus, D. G. Mixon, M. J. Poteet, and N. Strawn, "Constructing finite frames of a given spectrum and set of lengths," Applied and Computational Harmonic Analysis, vol. 35, no. 1, pp. 52-73, 2013.

[12] P. G. Casazza, A. Heinecke, F. Krahmer, and G. Kutyniok, "Optimally sparse frames," IEEE Transactions on Information Theory, vol. 57, no. 11, pp. 7279-7287, 2011.

[13] P. G. Casazza, M. Fickus, A. Heinecke, Y. Wang, and Z. Zhou, "Spectral tetris fusion frame constructions," The Journal of Fourier Analysis and Applications, vol. 18, no. 4, pp. 828-851, 2012.

[14] P. G. Casazza, A. Heinecke, K. Kornelson, Y. Wang, and Z. Zhou, "Necessary and sufficient conditions to perform spectral tetris," Linear Algebra and its Applications, vol. 438, no. 5, pp. 22392255, 2013.

[15] K. Dykema, D. Freeman, K. Kornelson, D. Larson, M. Ordower, and E. Weber, "Ellipsoidal tight frames and projection decompositions of operators," Illinois Journal of Mathematics, vol. 48, no. 2, pp. 477-489, 2004. 


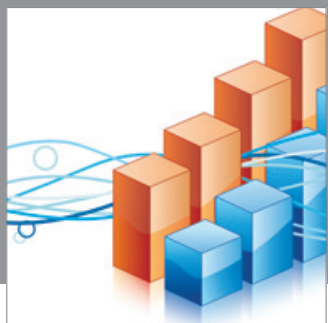

Advances in

Operations Research

mansans

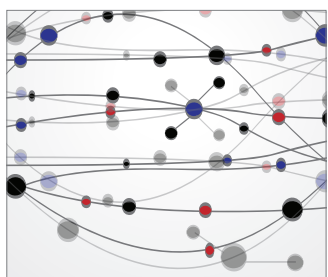

The Scientific World Journal
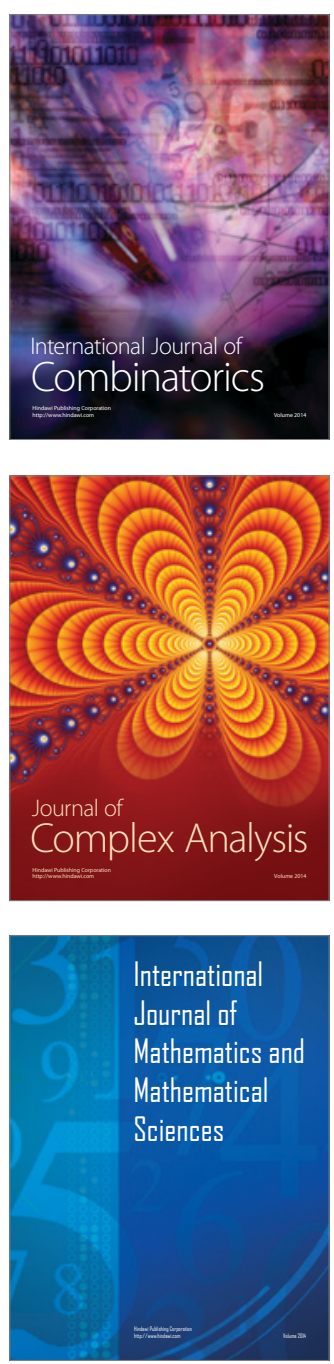
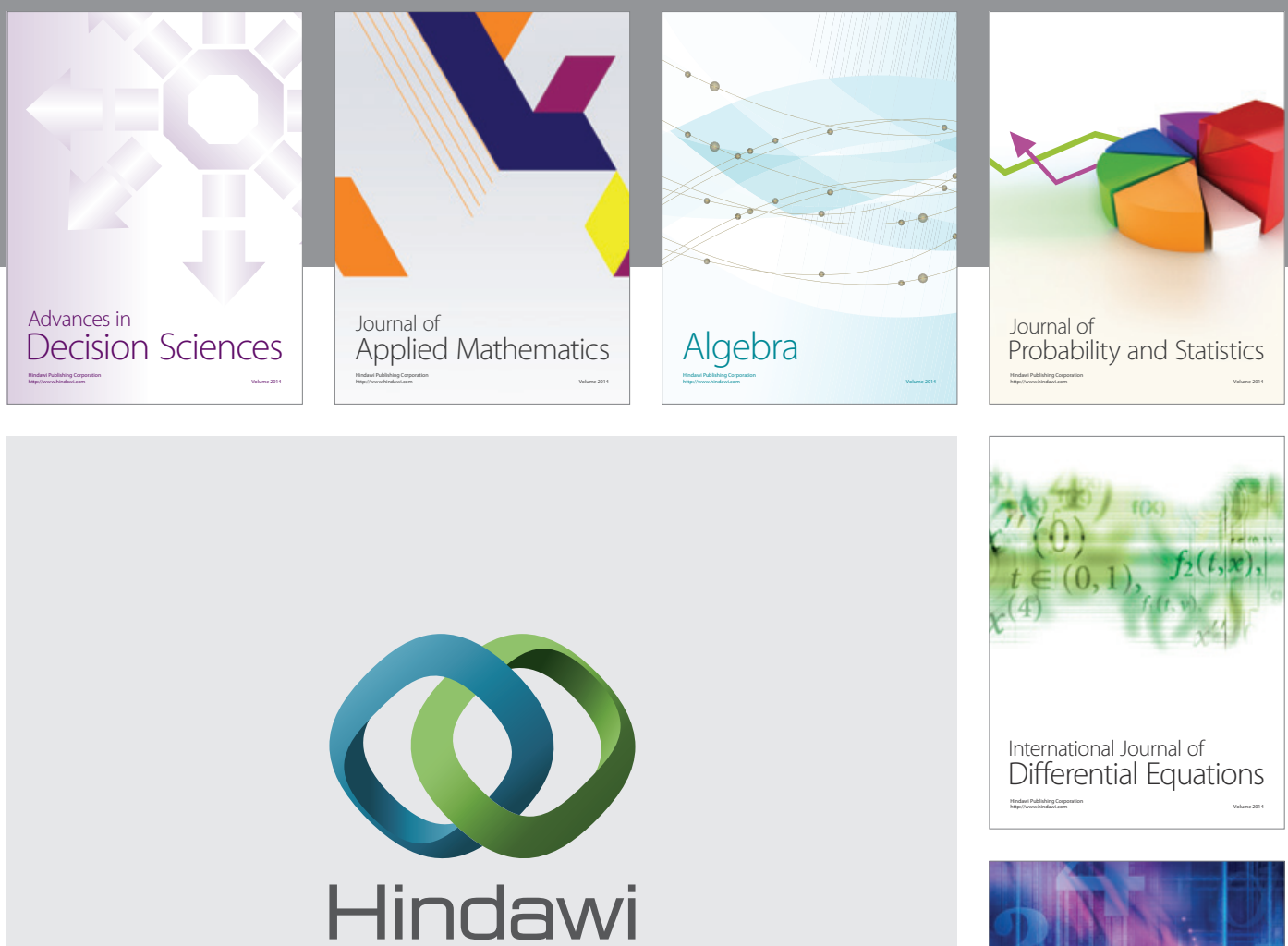

Submit your manuscripts at http://www.hindawi.com
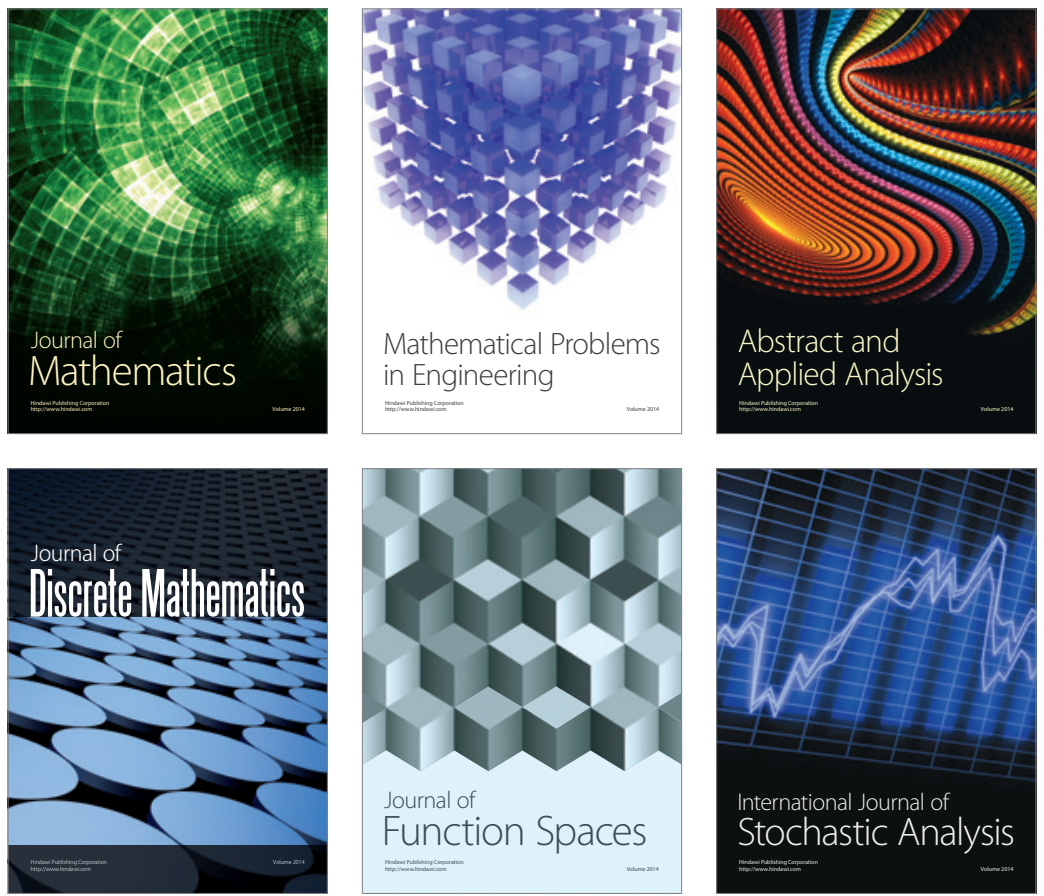

Journal of

Function Spaces

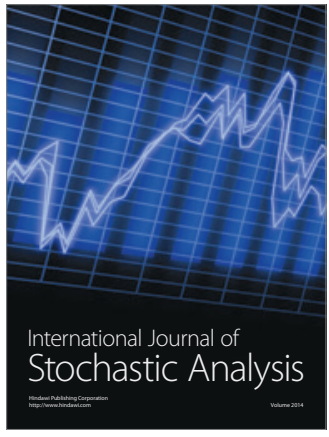

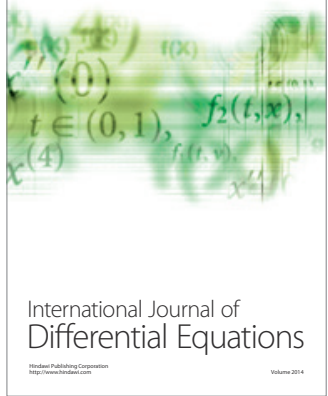
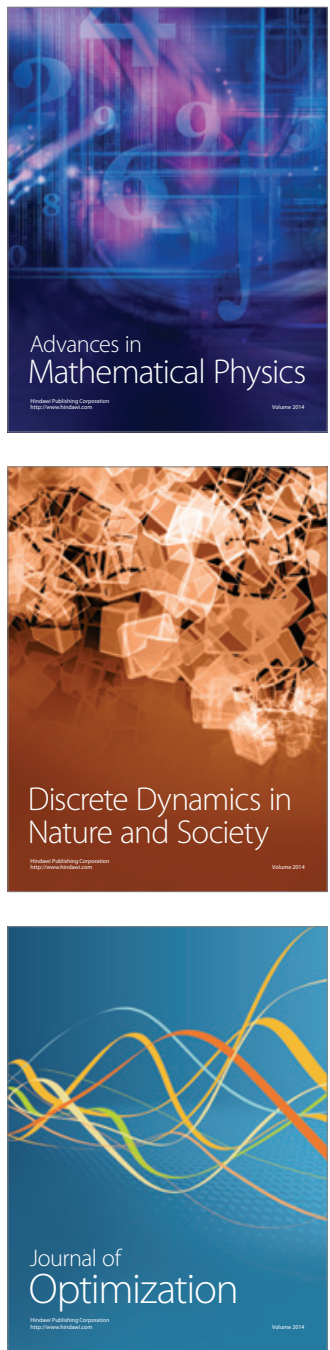